\title{
Immunohistochemical expression of endothelial cell markers in case of Dieulafoy lesion: Is there is a differences?
}

\author{
Julian Ananiev', Mariana Penkova ${ }^{2}$, Kiril Slaveykov ${ }^{3}$, Georgi Tchernev ${ }^{4}$ \\ ${ }^{1}$ Department of General and Clinical Pathology and Forensic Medicine, Medical Faculty, Trakia University, Stara Zagora, Bulgaria \\ ${ }^{2}$ Department of Internal medicine, Medical Faculty, Trakia University, Stara Zagora, Bulgaria \\ ${ }^{3}$ Department of Ophthalmology and General medicine, Medical Faculty, Trakia Univercity, Stara Zagora, Bulgaria \\ ${ }^{4}$ Saint Kliment Ohridski University, Medical Faculty, University Hospital Lozenetz, Sofia, Bulgaria
}

\section{Email address:}

julian.r.ananiev@gmail.com (J. Ananiev),mpenkovadoc@abv.bg (M. Penkova),kirilslaveykov@gmail.com (K. Slaveykov), georgi_tchernev@yahoo.de(G. Tchernev)

\section{To cite this article:}

Julian Ananiev, Mariana Penkova, Kiril Slaveykov, Georgi Tchernev. Immunohistochemical Expression of Endothelial Cell Markers in Case of Dieulafoy Lesion: Is There Is a Differences?. Science Journal of Clinical Medicine. Vol. 3, No. 4, 2014, pp. 70-74. doi: $10.11648 /$ j.sjcm.20140304.13

\begin{abstract}
Dieulafoy's lesion (DL) is a rare of cases of acute upper GIT bleeding. We investigate the immunohistochemical expression of several endothelial markers in a patient with Dieulafoy's lesion of the stomach, by comparing the results with data on the expression of these markers in other types of gastric pathology. The histological examination of the patient with Dieulafoy's lesion showed small erosive defect covered with fibrin, under which there were several highly deformed and dilated blood vessels with different diameters. We found that the expression of EC markers $\mathrm{CD} 31, \mathrm{CD} 34, \mathrm{vWF}$ and VEGF in gastric blood vessels of DL is heterogeneous and there is some differences compared with endothelial markers expression in other gastric pathology.
\end{abstract}

Keywords: Gastric Blood Vessels, CD31, CD34, Von Willebrand Factor, VEGF

\section{Introduction}

Dieulafoy's lesion (DL) is a rare pathology of the gastrointestinal tract (GIT), characterized by an aberrant submucosal arterial vessel, surrounded by intact or damaged mucosa that can lead to massive bleeding and life-threatening condition (1). From the pathological point of view, the process occurs most frequently in the stomach, followed by the duodenum, colon/rectum and other parts of the GIT, while histologically it is presented as a small defect in the mucosa, surrounded by fibrinoid necrosis, on the bottom of which there is an abnormally dilated arteries $3 \mathrm{~mm}$ in diameter $(2,3)$.

Like other arterial vessels, arteries in DL are covered on the inside with a layer of endothelial cells (EC), having a significant number of functions and taking part in processes such as coagulation, thrombolysis, permeability, inflammation, tissue repair, angiogenesis, and others. At the same time it is known that the EC of various vessels in the human body have varying ability to express endothelium specific markers (4).

Factor VIII- related antigen, or von Willebrand factor (vWF) is a glycoprotein synthesized by endothelial cells and megakaryocytes. Its function in the body is two-fold: on the one hand, together with other proteins it forms the antihemophilic factor, on the other it is an activator of factor $\mathrm{X}$ in the clotting system, while also playing a major role in platelet aggregation (5). Although it is a specific vascular marker, different vessels may show variable expression of vWF $(6,7)$.

CD31 or platelet-endothelial cell adhesion molecule- 1 (PECAM- 1) is another endothelial marker that can be expressed by platelets and megakaryocytes, and its role is associated with participating in cell adhesion and angiogenesis (8).

Another widely investigated endothelial growth factor is a human CD34 or a hematopoietic progenitor cell antigen 
that is expressed on a number of vessels, but also by the tumor cells in many mesenchymal tumors. A number of authors report heterogeneous expression, making it partially applicable for classification of certain diagnoses (9).

Vascular-endothelial growth factor (VEGF) is a heparincontaining angiogenic growth factor demonstrating high specificity for endothelial cells, while VEGF-A is one of the regulatory factors for both the physiological and pathological angiogenesis (10).

We aimed to investigate the immunohistochemical expression of several endothelial markers in a patient with Dieulafoy's lesion of the stomach, by comparing the results with data on the expression of these markers in other types of gastric pathology.

\section{Materials and Methods}

\subsection{Patients}

Dieulafoy's lesion (DL) is a rare pathology of the gastrointestinal tract (GIT), characterized by an aberrant submucosal arterial vessel, surrounded by intact or we selected a patient with Dieulafoy's lesion of the stomach, which after careful microscopic examination was prepared for histological examination and stained with hematoxylin/eosin. The diagnosis was confirmed by two pathologists independently from one another.

To compare the expression of various endothelial markers, we used archival cases of patients with: Intestinal type of gastric cancer, chronic gastric ulcer, and chronic gastritis associated with H.pylori.

\subsection{Immunohistochemistry}

The samples were fixed in $10 \%$ formalin, included in paraffin and cut in $5 \mathrm{mkm}$ shears. After deparaffination shear, the blocking of the endogen peroxidase is accomplished according to the protocol in the frames of 5 min with blocking the peroxidase agent. Three times jet washing with buffer was following and incubation with initial antibody for $1 \mathrm{~h}$. After the following three times washing, the chemicals were incubated with marked polymer for $20 \mathrm{~min}$ and again were washed. In the last phase the chemicals were incubated with DAB Substratechromogen were jet washed again and at the end were contra stained hematoxylin of Mayer.

There were used the following components for immunohistochemical examination: antibodies - vWF (M0616, DAKO, Denmark) in dilution 1:50, CD31 (M0823, DAKO, Denmark) in dilution 1:40, CD34 (NCLL-END, Leica Biosystems, UK) in dilution 1:100, VEGF (M7273, DAKO, Denmark) in dilution 1:50 and detection system EnVision ${ }^{\mathrm{TM}}$ FLEX+, Mouse, High pH, (Link) (K8002, DAKO, Denmark). Immunehistochemical examination was accomplished according to the protocols of the production companies.

\section{Results}

The histological examination of the patient with Dieulafoy's lesion showed small erosive defect covered with fibrin, under which there were several highly deformed and dilated blood vessels with different diameters (Fig. 1).

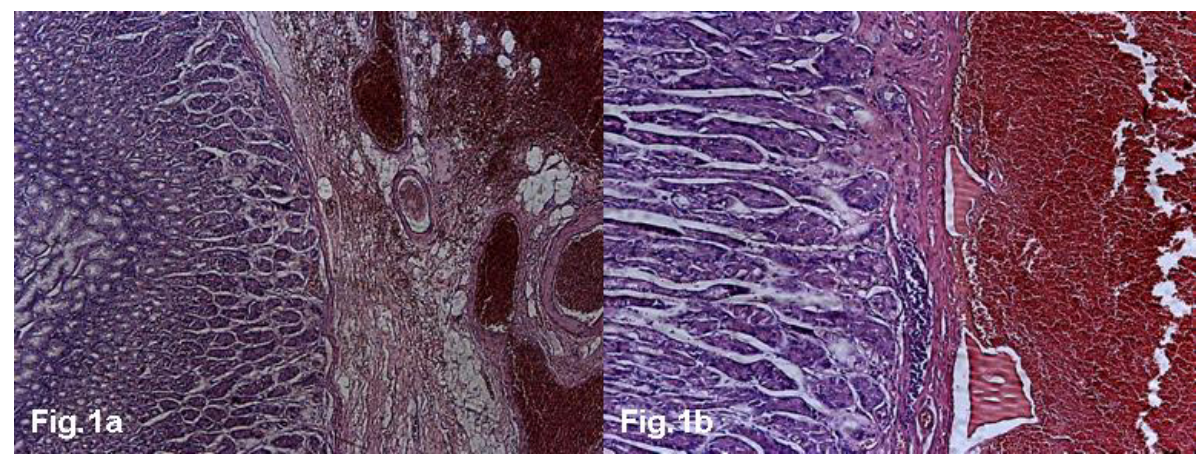

Figure 1 a, b. Histological examination of Dieulafoy's lesion /x50,x100/. 


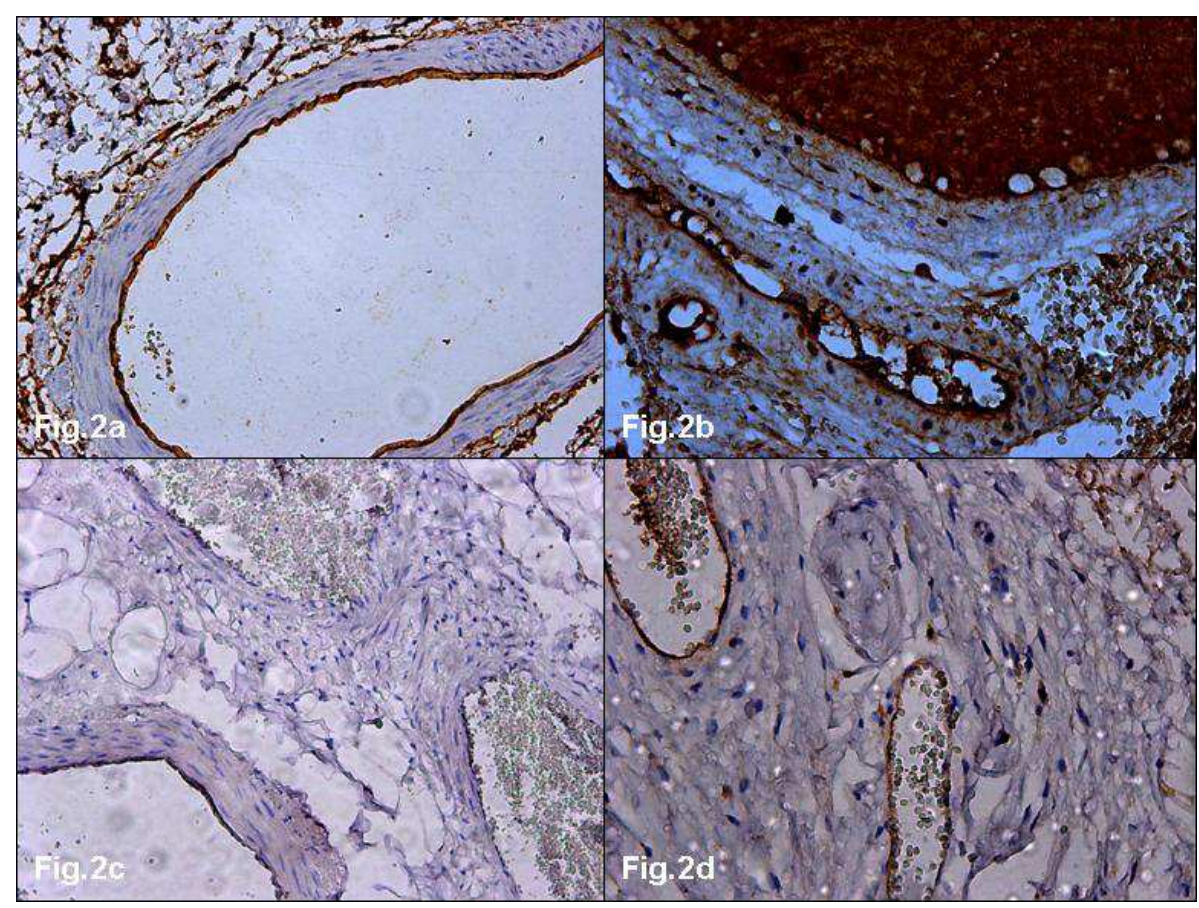

Figure 2. Dieulafoy's lesion expression of: a. CD31-strong ; b. von Willebrand Factor-strong; c. CD34-negative; d. VEGF-moderate. /x200/.

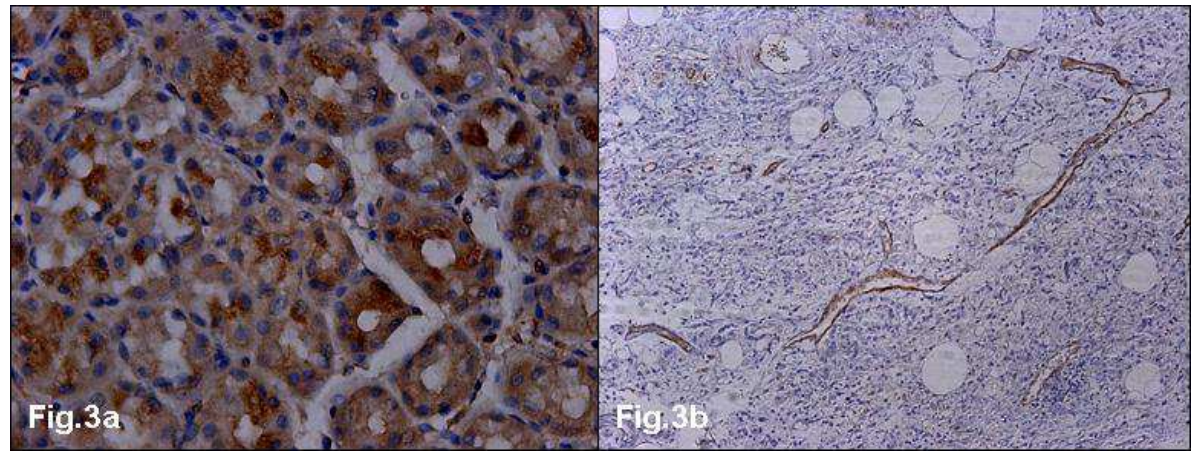

Figure 3. a. Expression of VEGF-positive normal epithelium cells/x400/; b. expression of VEGF-positive vessels in gastric cancer specimen /x100/.

After the immunohistochemical analysis varying degrees of expression of the tested markers were found (Table 1a,b).

In the endothelial cells of the patient with DL we observed a strong expression of CD31 and vWF, and a moderate expression of VEGF. CD34 marker was not observed in the examined case (Figure 2a-d). In the other cases all three markers were expressed: CD31, CD34 and vWF, while VEGF expression was observed in normal gastric epithelium, in vessels and tumor cells of gastric carcinoma but not in normal gastric vessels (Figure 3a,b). In addition, the vessels of the carcinoma had a lower level of expression of the markers. In the case of chronic gastritis associated with H.pylori a weak expression of VEGF was observed in single EC of normal mucosa vessels.
Table 1. Comparative degree of expression of EC markers in the various lesions.

\begin{tabular}{|c|c|c|c|c|}
\hline Lesion & CD31 & CD34 & vWF & VEGF \\
\hline $\mathrm{DL}$ & +++ & - & +++ & ++ \\
\hline Gastric cancer & ++ & ++ & ++ & - \\
\hline $\begin{array}{l}\text { Chronic ulcer } \\
\text { H.pylori }\end{array}$ & +++ & +++ & +++ & - \\
\hline $\begin{array}{l}\text { associated } \\
\text { gastritis }\end{array}$ & +++ & +++ & +++ & $+?$ \\
\hline
\end{tabular}

\section{Discussion}

In the investigated rare case of Dieulafoy's lesion, we found a high expression of three of the four endothelial markers used. For comparison we used the control tissues from patients with various diseases and compared the level of expression.

There are hypotheses that despite their identical origin EC in different vessels may express different vascular 
markers. A typical example is the expression of the marker D2-40, characteristic of lymphatic vessels, which is not expressed by the endothelium of arteries and veins, and vice versa - markers, such as CD31, CD34 and vWF were expressed weakly or not at all in lymphatic endothelium (9).

This is also demonstrated in other in vitro studies, when in phenotypically distinct lines of endothelium a reduction of the expression of $\mathrm{CD} 31$ or CD34 markers is observed (4).

Of particular interest is the expression of VEGF in the DL and gastric cancer, as well as low expression in H.pylori associated gastritis. It is known that VEGF is secreted by the neoplastic cells in a variety of tumors, and is also found, although in a lower degree of expression, in the EC of their forming vessels $(11,12)$. It is the reason for tumor neoangiogenesis, and the fact that it is present in the EC can be explained by the proximity of the tumor cells secreting it and the paracrine regulation. Understanding mechanisms of gastric cancer angiogenesis provides a basis for a knowledge to the development of an antiangiogenic therapy for these patients. Other information in the publication of Pousa and Gisbert demonstrates that in patients with H.pylori associated chronic gastritis, elevated levels of VEGF in the gastric epithelial cells were detected, as well as by stimulating the activity of cyclooxygenase - 2 (COX-2) the levels of VEGF are also increased (13). Like paracrine effect on the endothelium in gastric cancer, a similar connection can be discussed here.

Interesting data we found in the article of Hudson et al. They investigate the relation between non-steroidal antiinflammatory drug (NSAID) therapy and gastroduodenal ulcer healing. The study therefore assessed the vascularity of 38 antral gastric ulcers immunohistochemically, using CD31 a vascular endothelial cell marker, in 17 patients taking NSAIDs and 19 control patients and the authors found that in the superficial granulation tissue NSAID therapy was associated with a significant reduction in the median number of capillaries (14). The authors also state that there were no differences in vascularity in the adjacent glandular mucosa and impairment of angiogenesis may be an important mechanism of NSAID related delayed ulcer healing.

The question remains - how can the expression of VEGF in patients with DL be explained, when it lacks any H.pylori infection, gastric cancer or precursor lesion? There is literature data available about the significance of this marker in a variety of tumor and inflammatory diseases of the stomach, but its relationship with malformation changes has not been established. Considering the rare pathology and the possibility for the expression to be characterized as a "chance finding", another hypothesis can be built on the basis that similarly malformed vessels are likely to express markers not typical for most normal EC. This hypothesis can explain the missing expression of CD34 marker in the same lesion. Presence or absence of expression is associated with various functions performed by EC in different places.
The level of expression of the endothelial markers CD31, CD34, von Willebrand Factor and VEGF in various tissues confirms the importance of their phenotypic characteristics during the formation of the vessels in the corresponding body parts. EC in different vessels either express or don't express a specific marker, which can be used to describe a specific vessel and look for connection with the progression or other parameters for monitoring diseases. Dieulafoy's lesion is rarely examined for expression of these markers, but carrying out immunohistochemical analysis, gives further features of the pathological process. The presence of expression of VEGF, raises the question of the possibility that certain "imperfect" or "modified" vessels produce a protein impacting neoangiogenesis.

\section{References}

[1] Choi YC, Park SH, Bang BW, Kwon KS, Kim HG, Shin YW. Two cases of ileal dieulafoy lesion with massive hematochezia treated by single balloon enteroscopy. Clin Endosc. 2012 Nov;45(4):440-3.

[2] Morowitz M, Markowitz R, Kamath B, Allmen D. Dieulafoy's lesion segmental dilatation of the small bowel: an uncommon cause of gastrointestinal bleeding. J Paediatr Surg 2004; 39: 1726-8.

[3] Marangoni G, Cresswell AB, Faraj W, Shaikh H, Bowles MJ. An uncommon cause of life-threatening gastrointestinal bleeding: 2 synchronous Dieulafoy lesions. J Paediatr Surg 2009; 44: 441-3.

[4] Muller AM, Hermanns MI, Skrzynski C, Nesslinger M, Muller KM, Kirppatrick J Expression of the endothelial markers PECAM-1, VWf and CD34 in vivo and in vitro. Exp Mol Pathol 2002a; 72:221-229.

[5] Ogawa K, Oguchi M, Yamabe H, et al. Distribution of collagen type IV in soft tissue tumors: An immunohistochemical study. Cancer. 1986;58:269-277.

[6] Weiss HJ. von Willebrand factor and platelet function. Ann NY Acad Sci, 1991; 614:125-137.

[7] Muller AM, Skrzynski C, Skipka G, Muller KM. Expression of vonWillebrand factor by human pulmonary endothelial cells in vivo. Respiration (Herrlisheim), 2002c; 69:526-533.

[8] Marelli-Berg FM, Clement M, Mauro C, Caligiuri G. An immunologist's guide to CD31 function in T-cells. J Cell Sci. 2013 Jun 1;126(Pt 11):2343-52.

[9] Pusztaszeri MP, Seelentag W, Bosman FT. Immunohistochemical expression of endothelial markers CD31, CD34, von Willebrand factor, and Fli-1 in normal human tissues. J Histochem Cytochem. 2006 Apr;54(4):385-95.

[10] Ferrara N. Vascular endothelial growth factor. Arterioscler Thromb Vasc Biol 2009;29:789-91.

[11] FerraraN,Davis-Smyth T. The biology of vascular endothelial growth factor. Endocr Rev 1997;18:4-25. 
[12] Dvorak HF, Sioussat TM, Brown LF, et al. Distribution of vascular permeability factor (vascular endothelial growth factor) in tumors: concentration in tumor blood vessels. J Exp Med 1991;174:1275-8.

[13] Pousa ID, Gisbert JP. Gastric angiogenesis and Helicobacter pylori infection. Rev Esp Enferm Dig. 2006 Jul;98(7):52741.
[14] Hudson N, Balsitis M, Everitt S, Hawkey CJ. Angiogenesis in gastric ulcers: impaired in patients taking non-steroidal anti-inflammatory drugs. Gut. 1995 Aug:37(2):191-4. 